\title{
A NOTE ON THE POSSIBLE EVOLUTIONARY STATUS OF THE RECURRENT NOVA T CRB
}

\author{
(Abstract) \\ PETR HARMANEC \\ Ondřejov Observatory, Czechoslovakia
}

I would like to make a comment on the possible interpretation of the recurrent nova T CrB comparing this object with another one, namely with the shell star AX Mon. Basic characteristics of both binaries are:

$$
\begin{aligned}
& \text { AX Mon: B2 IV-Ve }+\mathrm{K} 2 \text { II, orbital period } P=232.5 \text { days } \\
& \text { T CrB: } \mathrm{M} \text { giant }+\mathrm{Be}, \quad \text { orbital period } P=230 \text { days, }
\end{aligned}
$$

total mass of both systems being probably comparable (depending on the assumed inclinations of orbits).

Some time ago I computed (originally in co-operation with Prof. Plavec) several sequences of models in a case B of mass exchange in an attempt to represent the present stage of AX Mon. I found that for any reasonable combination of initial masses the mass-losing component had to have a deep outer convective zone at the very beginning of mass exchange. It was shown by Paczyński that in such a case mass exchange must proceed on the dynamical time scale of the outer envelope, the radius of the star remaining nearly constant while the absolute dimension of the Roche lobe is shrinking as long as the role of both components is not reversed. Only after that the mass exchange can goes on the Kelvin time scale of the mass-losing star.

Four years ago Bath suggested this instability may be responsible for the nova phenomenon. I think he might be right at least for the case of $\mathrm{T} \mathrm{CrB}$. It is noticeable that in $\mathrm{T} \mathrm{CrB}$ the $\mathrm{M}$ giant is the more massive component of the system being thus dynamically unstable while AX Mon has the Be-component more massive - being according to my model just at the end of the dynamically unstable phase i.e. only thermally unstable now.

Another fact supporting the principal correctness of Bath's theory is the low limit of the mass of the Be component of $\mathrm{T} \mathrm{CrB}$ which exceeds the white-dwarf limit.

Thus, I think, a direct evolutionary connection may exist between novae of $\mathrm{T} \mathrm{CrB}$ type and some shell stars which both are - as I believe - binaries in the stage of rapid mass exchange between components.

\section{References}

Horn, J. and Harmanec, P.: 1971, paper presented at the Czechoslovak Conference on Stellar Astronomy and Astrophysics, Cikháj, October 12-14, 1971 (see also: Folia Fac. Sci. Nat. Univ. Purkynianae Brunensis 14, ser. Physica, No. 2, p. 71, 1973).

Harmanec, P.: 1974, Submitted for publication in Bull. Astron. Inst. Czech. 25.

Plavec, M., Ulrich, R. K., and Polidan, R S.: 1973, to be published in Publ. Astron. Soc. Pacific. 